\title{
TECHNOLOGY IN THE AVIATION METEOROLOGY CLASSROOM: A PILOT STUDY
}

\author{
Michael R. Witiw \\ Phillip Horton \\ Florida Institute of Technology
}

\begin{abstract}
Over the past few years, advanced technology that provides interactive and current weather data has become available in the aviation meteorology classroom. As part of its Airway Science Program, the Federal Aviation Administration has helped finance the installation of this technology at colleges and universities with aviation programs. The new systems were to be used in a dual role, as both an instructional aid and a flight planning tool. This study explores the efficacy of the technology as an aid to instruction by comparing students who had access to the technology to those who did not. The technology was used to provide relevant background material on a topic before the topic was formally presented in the course (a term frequently used to describe such background material is 'advance organizer'). Next, in order to statistically match study subjects, predictor variables for success in a basic aviation meteorology course were determined. Last, a pilot study was conducted with students who had access to the technology, comparing their success to those who did not. The encouraging results of this pilot study were reported to the FAA in an institutional Airway Science grant report.
\end{abstract}

\section{Introduction}

The past several years have seen the introduction of technology into the meteorology classroom (Wash et al., 1992; Byrd, DeSouza, Fingerhut, \& Murphy, 1994). This technology has basically taken two forms. Many of the large universities that offer an academic major program in meteorology use the Unidata system, a University Corporation for Atmospheric Research (UCAR) program funded by the National Science Foundation. This system basically takes meteorological data provided through the Internet and processes that information using one of several software packages. The software then allows for different types of interactive meteorological data analyses using micro-computers. Examples of the software packages include the McIdas system developed at the University of Wisconsin and the WXP system developed at Purdue University (Wash, et al., 1992). Typically, these software packages would be used by students studying synoptic or mesoscale meteorology. For less advanced students in a survey course, different meteorological products can be looped into an animation display (Wash et al., 1992).

The second type of system in use is provided by one of several commercial vendors (Kavouras, Inc. provided the technology used in this study), and allows for a certain amount of interactive work. Its main strength, however, lies in the wide variety of raw analyzed data that are available. The data provided are more timely than that provided by the UNIDATA system, therefore this system meets the dual needs of the aviation student. That is, it not only serves as an aid to learning meteorology as an academic discipline, but can also serve as an effective flight planning tool. These systems are popular with the Federal Aviation Administration (FAA), and, as a result, have been placed in several collegiate aviation programs. 
During the 1993 Unidata workshop meeting, a roundtable discussion group addressed the efficacy of the available technology in improving learning among students. Although the general feeling was that it did improve learning, no specific examples were given (Byrd et al., 1994). A large capital investment both on the part of the FAA and the recipient university is required, therefore it would seem appropriate to investigate the efficacy of the new technology in aiding the leaming of meteorological concepts by aviation students and the value of these investments.

This study was comprised of three parts. Initially, a theoretical framework to help explain why this technology should be effective as a learning tool and how best to use it was explored. Secondly, suitable predictors for a student's success in an aviation meteorology course were determined. That was a necessary step so that the study's outcome could be controlled for the influence of extraneous variables. Lastly, the predictors were used in a limited, controlled, causal-comparative study in order to quantify any effects this new technology may have on success in learning meteorology.

\section{The Role of Prior Knowledge}

In the early 1960s, the educational researcher, Ausubel (1960), began exploring the role of prior knowledge. The theory that prior knowledge can influence learning can be traced at least as far back as the turn of the century and the philosopher Herbart, but it is with the work of Ausubel that research into this area really began (Bames \& Clawson, 1975). Ausubel used the terms "advance organizer" and "assimilation theory" to help explain his ideas. In an experiment described in 1960, Ausubel saw strong evidence that a written advance organizer containing general and abstract ideas influenced leaming of a subsequent passage better than a historical overview of similar length (Ausubel, 1960). As quoted in Mayer (1979), advance organizers are "appropriately relevant and inclusive introductory materials...introduced in advance of learning... and presented at a higher level of abstraction, generality and inclusiveness." Ausubel talks of the "ideational anchorage" provided by ideas already present in a student's cognitive structure (Ausubel, 1980). Assimilation theory involves the idea of new knowledge being related to existing ideas already present. The new knowledge is then more readily understood. Lawton and Wanska (1977) elaborated: "Existing cognitive structure is the most crucial factor influencing new learning."

Although the advance organizers described by Ausubel (1960), Mayer (1979) and Lawton and Wanska (1977) were generally written organizers, Lawton and Wanska mention visual and audio organizers as well. Kenny (1993), in discussing computer based instruction, also talks of graphic organizers and pictorial graphic organizers.

The concept of an advance organizer is not, however, without its critics. Anderson, Spiro, and Anderson (1978), for example, felt Ausubel conducted his experiments when the concept of cognitive structures in terms of schemata was still in its developing stage. Their critique continued: "... when the reader does not possess relevant schemata, there is no reason to suppose they can be acquired from a few abstractedly worded sentences." (p. 439). In a response to his critics, Ausubel defended his ideas, and explained why organizers are dependent upon the material and that their specific structure varies (Ausubel, 1978).

The idea of advance organizers seems to be appropriate for use in a basic meteorology course where advanced ideas such as frontal systems and air masses can be presented early using technology in a general and abstract way, before their detailed discussion in the classroom. It is thought these advance organizers will help develop relevant schemata (or cognitive structures) 
in the students for the detailed concepts that follow. Mayer (1979) feels advance organizers work best with technical and unfamiliar ideas, therefore, this would appear to be an ideal situation for their use.

\section{Statistical Matching on Extraneous Variables}

Before the effectiveness of an instructional method can be assessed, and where random assignment of subjects is not practical, the problem of controlling for extraneous variables must be addressed. In a causal-comparative study such as this, statistical matching on these extraneous variables is a recommended way to control for their influence (Fraenkel \& Wallen, 1993). Initially for this study, Scholastic Apptitude Test (SAT) scores, Grade Point Averages (GPAs), and high school class standings were considered the variables on which to match subjects in the control and treatment groups.

Recently, several studies have attempted to show that SAT scores add very little to predicting the subsequent performance of college students. A study by Baron and Norman (1992) looked at total SAT scores, high school class rank, and College Board achievement tests scores as predictors of college cumulative grade point averages. The study looked at freshmen who entered the University of Pennsylvania in 1983 and 1984. When the SAT scores were entered as a single zero-order variable, they accounted for only .04 of the total variance. When entered last in a hierarchical regression with achievement test scores and class standing, they contributed nothing to the total variance. Examination of the quadratic term did not change the results.

In recent years, the SAT's ability to predict college performance has apparently decreased. A study by Morgan (1989) reviewed 778 validity studies from 222 colleges accomplished from 1976 to 1985 . Correlation of SAT scores with first year grades gradually decreased from .51 to .47 over this period (Morgan, 1989). Investigation as to whether this was caused by a change in the SATs that took place in 1985 was completed by Stricker (1991). By correlating the 1975 and 1985 SATs to class rank and high school GPA, he concluded that the 1985 SATs were no less valid than their 1975 predecessors. While Stricker sees the correlation of SAT scores with high school GPA and class standing as confirming the SATs' validity, Crouse and Truesheim (1991) see this as a redundancy. Because of this, they feel very few admissions decisions are changed because of SAT scores, and that the benefits of the SATs are small to non-existent. On the other hand, a study conducted at California State University (Hayward) found that SAT scores added a small but significant increase in ability to predict freshmen year GPAs (Cowen \& Fiori, 1991). Kanoy, Wester, and Latta (1989) concluded that SATs and GPA were good predictors for high level students, explaining more than 50 percent of the variance when predicted GPA was 2.9 or higher but did not work well for students with low potential. Overall, these studies come to mixed conclusions as to whether the SATs have an additive effect in predicting college success as determined by GPA. Despite this controversy, there appears to be enough evidence that SAT scores should at least be considered as matching extraneous variables in a specific college course.

\section{Methods}

In the aviation program of one university, and probably many others, aviation meteorology is a required course for all entering students. It is usually completed during the first semester of the first year. The students enrolled in this course during the Fall 1993 semester provided a 
control group and a source to statistically match subjects. Well into this semester, the new technology, produced by Kavouras, Inc., was installed, and was comprised of a METPAC system which provides hard copy graphical and alpha-numberic data as well as a TRIMETS system which displays looped graphical, radar, and satellite data. Installation was partially funded by an FAA Airway Science grant. Although some use of the new systems was made during the latter half of the semester, initial startup problems and time constraints proved inadequate to implement their routine and systematic use. By the spring semester of 1995, a systematic approach to the use of the new systems was in place and appeared to be working well. The first eight weeks of the course, which included the period during which the control group had no access to the new systems, in contrast to the experimental group, was the main focus of the study.

\section{Control Group Population and Subjects}

During Fall 1993, four sections of aviation meteorology were offered with a total student number of 68 . Twenty-two of these students were enrolled in a section taught by an adjunct instructor. Those students were excluded from the current study because of lack of standardization of both teaching and testing methods between instructors. The remaining three sections were taught by the same instructor and included 46 students. Of those 46 students, 11 were excluded from this study because their SAT scores and high school GPAs were not available for various reasons. Transfer students, for example, typically did not have this information in their files. This reason accounted for 9 cases. In two other cases, the data were simply not present in the students' files. One student was excluded because his 200 score on the verbal portion of the SAT indicated his English skills were probably inadequate. This was reflected in a 42 average computed without the benefit of a final examination which he did not complete. Total subjects remaining as a control for this study were 34 . All were native English speakers, and 7 were female. Of these 34,3 had no high school class standing reported, but it was a relatively simple matter to account for this missing data using substitution of the mean.

\section{Experimental Subjects}

The subjects were five students enrolled in aviation meteorology in Spring, 1995. Because most students take this course in the fall semester, only 13 were enrolled in the Spring, 1995 course. Further, since many of these students were transfer students, SATs and high school GPAs were available for only six students. Additionally, one of these students became ill for the two weeks prior to the mid-term examination missing the lecture and class discussion of important material. All experimental subjects were male and native English speakers.

\section{Instruments}

The study was based on midterm examination results. The midterm was a 40 question instrument covering the same material for both the control and experimental groups. Thirty-one of the questions used in the experimental group's midterm were similar to, or variants of, the questions used in Fall, 1993. For the purpose of this study, only those 31 questions were graded. The instruments used in this study were group administered, criterion referenced, in-class examinations. The questions were instructor developed with some help from textbook support material. Types of questions comprising the total score and their portion of the total score were about $75 \%$ multiple choice, $15 \%$ fill in type questions (one or two words), as well as about $10 \%$ which required short sentences for answers. 


\section{Procedures}

The instrument was administered during the eighth week of the course. Each week prior to that time, a 25 minute laboratory period was used in addition to normal classroom time. The control group for which the predictors were based had no use of the new technology for the same time period during their semester. During this extra 25 minute period, a meteorological presentation of current conditions was made. Concepts such as fronts, air masses and upper level analyses were introduced four to six weeks before they were discussed in some detail in the lecture. Students understood that the material presented during this laboratory session was not to be tested, but that it might benefit them sometime in the future. The midterm was group administered by section. Fifty minutes were allowed for the midterm. Time appeared adequate with all students completing the instrument. The same room was used for administering all instruments.

\section{Data Analysis}

As a preliminary step to evaluate matching variables, multiple linear and curvilinear regression techniques were used to analyze the relation of the independent variables (Verb - verbal SAT scores, Math - math SAT scores, GPA - high school GPA, and CS - class standing) to an overall dependent variable, final grade, overall average in aviation meteorology. This was a comprehensive evaluation of the variables as predictors before they were applied more specifically to the mid-term grade only. This was done to ensure adequate testing of the selected matching variables. The initial analysis considered all components of an overall grade for the control group. Four instruments determined this grade. The mid-term was valued at $30 \%$ of the final grade and was comprised of 40 questions while the first and third instruments were each valued at 15 . The final examination (representing 40 percent of the final grade) had 78 questions. It was these same techniques that were then repeated for the mid-term examination only, where the dependent variable was strictly the grade received on the mid-term exam.

\section{Results}

\section{Descriptive Statistics}

Table $I$ depicts selected descriptive statistics for all linear variables where overall final grade is the dependent variable. Class standing (CS) is expressed in terms of the upper fraction of a student's high school class. For example, if a student were 10 th out of a class of 100 , the student's CS value would be .10 . Sample size was 34 . Table 2 describes the correlations between variables. 
Table 1

Descriptive Statistics

\begin{tabular}{|c|c|c|c|}
\hline & Mean & S.D. & Range \\
\hline Verb & 480.588 & 77.575 & 340.000 \\
\hline Math & 554.118 & 74.635 & 370.000 \\
\hline GPA & 3.301 & .380 & 1.680 \\
\hline CS & .235 & .160 & .660 \\
\hline Grade & 76.897 & 7.870 & 30.350 \\
\hline
\end{tabular}

Table 2

Pearson r correlations between linear variables

\begin{tabular}{|l|c|c|c|c|c|}
\hline & Verb & Math & GPA & CS & Grade \\
\hline Verb & 1.000 & & & & \\
\hline Math & .302 & 1.000 & & & \\
\hline GPA & -.049 & .092 & 1.000 & & \\
\hline CS & -.161 & -.088 & -.673 & 1.000 & \\
\hline Grade & .475 & .534 & .423 & -.330 & 1.000 \\
\hline
\end{tabular}

The low correlations between SAT scores and high school GPA and class standing are interesting. The values for this sample are considerably lower than those reported by Stricker (1991), which ranged from .39 to .54 . The current sample also indicates little redundancy between SAT scores and either GPA or CS. This is in contrast to the conclusions drawn by Baron and Norman (1992) in their study at the University of Pennsylvania.

\section{Inferential Statistics}

For this study alpha was set at .05 and power at .80 . Initially, curvilinear effects for all four independent variables were calculated. The zero order linear terms of three of the four independent variables (all but CS) were significant at the preset alpha level. Neither the quadratic nor the cubic terms added significance to the independent variables. Therefore, a hierarchical regression was completed using only linear terms. To provide the required power effect size for the hierarchical model was set quite high ( $\mathrm{f} 2=.411, \mathrm{R} 2=.29, \mathrm{R}=.54$ for four independent variables and $\mathrm{f} 2=.393, \mathrm{R} 2=.26, \mathrm{R}=.51$ for 3 independent variables - calculations made in accordance with Cohen \& Cohen, 1983). Math and verbal SAT scores were entered as a set, followed by High School GPA. Finally CS was added to the computation. Power exceeded .80 in all cases. (See Table 3). It was determined that the overall best regression fit was the hierarchical using three independent variables (Math, Verb, GPA). The addition of CS added little 
to $\mathrm{R} 2$, and increased the overall effect size required. A regression summary is included in Table 3. The resulting predictive equation for the final course grade was: Grade $=8.226+.039$ Verb +.040 Math + 8.427 GPA.

Table 3

Regression Summary

\begin{tabular}{|l|c|c|}
\hline \multicolumn{1}{|c|}{ Variable } & DF & Cumulative R2 \\
\hline SAT (Verb \& Math) & 2,31 & $.394^{*}$ \\
\hline GPA & 3,30 & $.557^{* *}$ \\
\hline CS & 4,29 & $.570^{* *}$ \\
\hline
\end{tabular}

${ }^{*} \mathrm{p}<.001$

$* * \mathrm{p}<.0001$

In preparation for the pilot study, a regression analysis using only the midterm examination grade was made for the same subjects under the same conditions and using identical independent variables. Results were similar to when the overall (final) grade was the dependent variable and are shown in Table 4 . The equation developed was:

Midterm Grade $=2.538+.043$ Verb +.047 Math + 7.351 GPA

Table 4

Regression Summary

\begin{tabular}{|l|c|c|}
\hline \multicolumn{1}{|c|}{ Variable } & df & Cumulative R2 \\
\hline SAT (Verb \& Math) & 2,31 & $.308^{*}$ \\
\hline GPA & 3,30 & $.420^{* *}$ \\
\hline
\end{tabular}

$$
\begin{aligned}
{ }^{*} p<.01 \\
{ }^{* *} p<.001
\end{aligned}
$$

\section{Results of the Pilot Study}

The results for individual students follow in Table 5. Standard deviation for the instrument was 10.2 . Interestingly, while student $A$ received a grade nearly one half a standard deviation lower than expected, students C, D, and E all exceeded their predicted grade by more than one standard deviation. 
Table 5

Student Performance

\begin{tabular}{|c|c|c|}
\hline Student & Predicted Grade & Actual Grade \\
\hline A & 88.6 & 82.3 \\
\hline B & 78.3 & 79.0 \\
\hline C & 76.6 & 90.0 \\
\hline D & 67.0 & 77.4 \\
\hline E & 58.5 & 69.3 \\
\hline
\end{tabular}

\section{Conclusions}

SATs were shown to be good matching variables for the sample of the 34 students that comprised the control group in this study. Additionally the linear regression of SAT scores combined with high school GPA showed significance as well as considerable power for predicting success in a basic aviation meteorology course (Table 3), and therefore were good extraneous variables upon which to match the subjects. When the computations were repeated for just the midterm exam, similar significance was demonstrated. For this particular experimental group, it does appear that technology may have made a positive difference.

\section{Limitations}

The five students in the experimental group of this causal-comparative study comprised an extremely small group, and it is apparent that no inferences can therefore be made. Moreover, this study, where the experimental group received a treatment more than a year after data were collected from the control group would tend to be prone to external validity threats including history and possible unintentioned experimenter bias.

\section{Recommendations For Future Research}

Despite the study's limitations, the fact that three of the five students in the experimental group showed an improvement of over one standard deviation over what was expected, coupled with the fact that there seems to be a need for some definitive research in this area as implied in the meteorological literature (Byrd et al., 1994) indicate a larger, more comprehensive experimental or quasi-experimental study should be completed.

Additionally, this study only addressed the efficacy of meteorological work stations as a specific type of learning tool, an advance organizer. Recommended additional areas of research would be to evaluate these work stations from other educational perspectives and also as flight planning tools. 


\section{References}

Anderson, R. C., Spiro R. J., \& Anderson, M. C. (1978). Schemata as scaffolding for the presentation of information in connected discourse. American Educational Research Journal, 15, 433-440

Ausubel, D. P. (1960). The use of advance organizers in the learning and retention of meaningful material. The Journal of Educational Psychology, 51, 267-272.

Ausubel, D. P. (1978). In defense of advance organizers: A reply to the critics. Review of Educational Research, 48, 251-257.

Ausubel, D. P. (1980). Schemata, cognitive structure and advance organizers: A reply to Anderson, Spiro and Anderson. American Educational Research Journal, 17, 400-404.

Barnes, B. R., \& Clawson, E. U. (1975). Do advance organizers facilitate learning ? Recommendations for further research based on the analysis of 32 studies. Review of Educational Research, 45, 637-659.

Baron, J., \& Norman, M. F. (1992). SATs, achievement tests, and high school dass ranks as predictors of college performance. Educational and Psychological Measurement, 52, 1047-1055.

Byrd, G., DeSouza, R., Hingerhut, W., \& Murphy, C. (1994). Integrating technology into the meteorological classroom: A summary of the 1993 northeast regional Unidata workshop. Bulletin of the American Meteorological Society, 75, 1677-1683.

Cohen, J., \& Cohen, P. (1983). Applied multiple regression/correlation analysis for the behavioral sciences (2nd ed.). Hillsdale, NJ: Lawrence Erlbaum Associates.

Cowen, S., \& Fiori, S. J. (1991). Appropriateness of the SAT in selecting students for admission to California State University, Hayward (ERIC Document Reproduction Service No. ED 343 934)

Crouse, J., \& Truesheim, D. (1991). How colleges can correctly determine selection benefits from the SAT. Harvard Educational Review, 61, 125-147.

Fraenkel, J. R., \& Wallen, N. E. (1993). How to design and evaluate research in education. (2nd ed.). New York: McGraw-Hill.

Kanoy, K. W., Wester, J., \& Latta, M. (1989). Predicting college success of freshmen using traditional, cognitive, and psychological measures. Journal of Research and Development in Education, 22, 65-70.

Kenny, R. F. (1993). The effectiveness of instructional orienting activities in computer-based instruction (ERIC Document Reproduction Service No. ED 362 172)

Lawton, J. T., \& Wanska, S. K. (1977). Advance organizers as a teaching strategy: A reply to Barnes and Clawson. Review of Educational Research, 47, 233-244.

Mayer, R. E., (1979). Can advance organizers influence meaningful learning? Review of Educational Research, 49, 371-383.

Morgan, R., (1989). Analyses of the predictive validity of the SAT and high school grades from 1976 to 1985 (ERIC Document Reproduction Services No. ED 315 442)

Stricker, L. J. (1991). Current validity of 1975 and 1985 SATs: Implications for validity trends since the mid-1970s. Journal of Educational Measurements, 28, 93-98.

Wash, C. H., Desouza, R. L., Ramamurthy, M., Andersen, A., Byrd, G., Justus, J., Edmon, H., \& Samson, P. (1992). Teaching with interactive computer systems: A report on the Unidata/COMET/STORM workshop on synoptic/mesoscale inst ruction. Bulletin of the American Meteorological Society, 73, 1440-1447. 